\title{
An outbreak of encephalomyelitis in the Royal Free Hospital Group, London, in 1955
}

\author{
Nigel Dean Compston \\ M.A., M.D., F.R.C.P. \\ Royal Free Hospital, London
}

\begin{abstract}
Summary
This paper is a précis of the report given by the staff of the Royal Free Hospital (Medical Staff of the Royal Free Hospital, 1957) of an epidemic amongst the staff of the Royal Free Hospital in 1955.
\end{abstract}

\section{Introduction}

In October, 1957, the Medical Staff of the Royal Free Hospital reported in the British Medical Journal an outbreak of an illness amongst the hospital staff in 1955. Between 13 July of that year and 24 November, 292 members of the medical, nursing, auxiliary medical, ancillary, and administrative staff were affected by the illness and, of these, 255 patients were admitted to hospital. Dr Ramsay (1978) has already stated that similar cases had occurred in the population of North West London before this outbreak and sporadic cases continued to occur after the outbreak (Ramsay and O'Sullivan, 1956).

The disease was referred to as an encephalomyelitis and when the term 'benign myalgic encephalomyelitis' was subsequently introduced it was felt that the disease was best described by that title. The author has not had the opportunity of continuing to study the disorder but he certainly does not feel that the term 'epidemic neuromyasthenia' adequately describes the disorder.

\section{Clinical material}

In the paper of 1957 the staff of the hospital described in detail the clinical manifestations of 200 cases whom they felt were adequately studied. Table 1 indicates the initial manifestations in these cases. The clinical picture was variable both in the time pattern of its progression and the severity of the symptoms. In some cases characteristic symptoms appeared early and in others later. The first few cases were labelled as glandular fever although it was appreciated from the very start that they were not true cases of infectious mononucleosis. This term was used so as not to cause alarm.
TABLE 1. Initial manifestations in 200 cases

\begin{tabular}{lrl}
\hline Symptoms & $n$ & $\%$ \\
\hline Headache & 154 & 77 \\
Sore throat & 127 & $63 \cdot 5$ \\
Malaise & 124 & 62 \\
Lassitude & 102 & 51 \\
Vertigo & 94 & 47 \\
Pain in limbs & 93 & $46 \cdot 5$ \\
Nausea & 81 & $40 \cdot 5$ \\
Dizziness & 67 & $33 \cdot 5$ \\
Stiff neck & 65 & $32 \cdot 5$ \\
Pain in back & 64 & 32 \\
Depression & 38 & 19 \\
Abdominal pain & 29 & $14 \cdot 5$ \\
Vomiting & 24 & 12 \\
Diplopia & 18 & 9 \\
Tinnitus & 8 & 4 \\
Diarrhoea & 8 & 4 \\
\hline
\end{tabular}

It became clear early on in the outbreak that there $\stackrel{\mathbb{D}}{\stackrel{2}{ }}$ was organic involvement of the central nervous $\overrightarrow{\overrightarrow{0}}$ system but there were nevertheless abnormal signs outside; most particularly there was lymphadenopathy and fever. Table 2 indicates the non-neurological manifestations of the disease. As was emphasized in the original publication, fever was not 3 marked but occurred in $89 \%$ of the patients. It did not correlate with the degree of malaise suffered by a large number of the patients.

Usually by the second or third week of the disease, there was objective evidence of involvement of the 음 central nervous system which appeared to be $>$ characteristic of the outbreak. Similar manifestations had been described in previous outbreaks and were $N$ found in subsequent outbreaks.

Cranial nerve lesions largely affected the ocular- $N$ motor, facial and vestibular nerves and their $\underset{\omega}{N}$ incidence is indicated in Table 3. Pupillary changes sometimes occurred but the fundi and visual fields 0

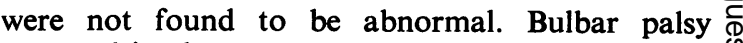
occurred in eleven cases.

Although there were sensory disturbances in the 7 limbs, motor disturbances were more common. 
TABle 2. Abnormal signs other than those in the central nervous system

\begin{tabular}{lrc}
\hline Signs & $n$ & $\%$ \\
\hline Severe injection of pharynx & 6 & 3 \\
Vesicles on pharynx & 6 & 3 \\
Enlarged cervical glands, ant. & 104 & 52 \\
Enlarged cervical glands, post. & 158 & 79 \\
Enlarged axillary glands & 73 & $36 \cdot 5$ \\
Enlarged epitrochlear glands & 20 & 10 \\
Enlarged inguinal glands & 65 & $32 \cdot 5$ \\
Neck rigidity & 22 & 11 \\
Subcostal tenderness (left) & 65 & $32 \cdot 5$ \\
Subcostal tenderness (right) & 64 & 32 \\
Palpable liver & 17 & $8 \cdot 5$ \\
Temperature range & 22 & 11 \\
Up to $98 \cdot 4^{\circ} \mathrm{F}\left(36 \cdot 9^{\circ} \mathrm{C}.\right)$ & 169 & $84 \cdot 5$ \\
$98 \cdot 6-100^{\circ} \mathrm{F}\left(37-37 \cdot 8^{\circ} \mathrm{C}.\right)$ & 2 & 1 \\
$100 \cdot 2-101^{\circ} \mathrm{F}\left(37 \cdot 9-38 \cdot 3^{\circ} \mathrm{C}.\right)$ & 7 & $3 \cdot 5$ \\
$101 \cdot 2^{\circ} \mathrm{F}+\left(38 \cdot 4^{\circ} \mathrm{C}.\right)$ & & \\
Pulse range & 91 & $45 \cdot 5$ \\
$60-80$ & 92 & 46 \\
$80-100$ & 17 & $8 \cdot 5$ \\
$100+$ & & \\
\hline
\end{tabular}

TABLE 3. Incidence of cranial-nerve lesions (148 cases)

\begin{tabular}{lrr}
\hline & Cases & $\%$ \\
\hline Cranial nerves & 69 & 46 \\
Ocular & 63 & 43 \\
Trigeminal & 6 & 4 \\
Facial & 28 & 19 \\
Eighth & 63 & 42 \\
Bulbar & 11 & 7 \\
Optic & 1 & 0.7 \\
Olfactory & 1 & 0.7 \\
\hline
\end{tabular}

There were varying degrees of paraesthesiae, however the most characteristic symptom was the prolonged painful muscle spasms that seemed to justify the use of the term 'myalgic' in the diagnostic label. Regrettably, photographs of a foot in spasm are no longer available. Painful spasms were invoked by passive movement. Their distribution was mixed and might be hemiplegic, paraplegic or monoplegic. Dr Richardson will describe the EMG findings which were matched clinically by a jerkiness of voluntary movement against resistance.

Involuntary movements, however, occurred spontaneously. Bladder dysfunction occurred in more than $25 \%$ of all the patients. Reflex changes were not pronounced but sometimes the reflexes were diminished and, on other occasions, exaggerated. The plantar responses were often equivocal and in only two cases clearly extensor. Muscle wasting was rare.

In summary, therefore, the limbs exhibited a combination of both irritative and paretic phenomena. The limb and trunk lesions are indicated in Table 4.

Amongst so many patients in a closed community suffering from a disease of unknown prognosis it would not be surprising if some patients exhibited extreme emotional or even hysterical features.

The course of the disease was variable and Table 5 indicates the duration of hospital admission. In

TABLE 4. Incidence of motor and sensory signs in limbs and trunk (148 cases)

\begin{tabular}{lcc}
\hline & Cases & $\%$ \\
\hline Limbs and trunk & 144 & 97 \\
Abnormal tendon reflexes & 122 & 76 \\
Motor weakness & 102 & 69 \\
Sensory disturbances & 82 & 55 \\
Muscle spasm & 18 & 12 \\
Myoclonus & 5 & $3 \cdot 4$ \\
Micturition disturbances & 39 & 26 \\
\hline
\end{tabular}

TABLE 5. Duration of hospital in-patient treatment

\begin{tabular}{lrc}
\hline & $n$ & $\%$ \\
\hline$<1$ month & 114 & 57 \\
$1-2$ months & 57 & $28 \cdot 5$ \\
$2-3$ months & 15 & $7 \cdot 5$ \\
$\geqslant 3$ months & 14 & 7 \\
\hline
\end{tabular}

many patients the symptoms waxed and waned in intensity over a long period. In the very large majority of cases the recovery of neurological function was complete and at the time of the staff's original publication almost two years after the outbreak only four patients still presented marked physical disability. A small number manifested prolonged depression and one patient committed suicide owing to her concern over a diagnosis of multiple sclerosis which had been made before the attack of encephalomyelitis.

\section{Investigations}

Special investigations did not reveal any specific abnormalities. Cerebrospinal fluid examined in a number of the earlier cases was normal. Haematological studies revealed abnormal lymphocytes of the type found in a number of virus diseases. The ESR was not usually raised. There were no characteristic biochemical changes. Post-mortem material was subsequently available from a patient with carcinoma of the ovary and of the patient already referred to who committed suicide. No changes attributable to the disease were found. The diagnosis of multiple sclerosis in the second case was confirmed. 
The epidemiological features of the outbreak were reported by Crowley, Nelson and Stovin in 1957. It is not surprising that there was a much higher incidence of the disease amongst resident than non-resident members of the hospital staff. The resident population was predominantly female giving, of course, a marked increase of incidence amongst female patients. The number of resident males is insufficient to produce adequate comparisons. There were only twenty at risk as against $\mathbf{8 4 0}$ females. The incidence of the disease however was similar in both groups. Over all, taking staff, students and patients, the incidence amongst males was $2 \cdot 8 \%$ as against $10.4 \%$ in females. However, if residents only are included, the incidence in males falls to $0.8 \%$ as against $11 \%$ of those exposed; of necessity there was a need to admit residents more often than nonresidents.

Needless to say, extensive investigations done in the hospital and many other institutes failed to reveal an aetiological agent of an infective nature. A toxic cause for the condition was investigated but no positive results were found.

It is impossible to conclude this presentation without commenting upon the papers of McEvedy and Beard in 1970. One is entitled 'Royal Free epidemic of 1955: a reconsideration' and the other 'Concept of benign myalgic encephalomyelitis'. In the first of the papers, the authors, following the analysis of case notes, conclude that epidemic hysteria is a 'much more likely' explanation of the illness than that the disease was organic. The data that they consider support this hypothesis are the high attack rate in females; the intensity of the malaise compared with the slight pyrexia; the presence of subjective features similar to those seen in a previous epidemic of hysterical overbreathing; the glove-and-stocking distribution of the anaesthesia; and the normal findings on special investigations. Dr Richardson will no doubt comment on the ability of one of the authors to simulate the jerking movement of the limbs as was produced in the Royal Free disease (Richardson, 1956). As Dr Ramsay has said, this outbreak occurred in the middle of a more sporadic outbreak in North West London which had started before the Royal Free outbreak and continued after it. Case to case contact between patients and their relatives also occurred. McEvedy and Beard's conclusions ignore the objective findings of the staff of the hospital of fever, lymphadenopathy, cranial palsies and abnormal signs in the limbs. There are many infective disorders in which malaise and fever are not $\stackrel{\varrho}{c}$ correlated. Overbreathing produces but transient sensory and motor phenomena. A glove-and- $\stackrel{\bar{s}}{+}$ stocking distribution of the anaesthesia occurs in ato number of organic diseases of the central nervous $\underline{ }$ system, e.g. in the peripheral neuropathies. Again, $\frac{\bar{\omega}}{\bar{D}}$ normal findings in the CSF are by no means in- $\stackrel{\mathbb{Q}}{\alpha}$ compatible with organic disease of the CNS and a normal ESR is frequently to be found in infectious mononucleosis.

These authors studied by questionnaire a number $\overrightarrow{\vec{H}}$ of the staff who suffered from the disorder, whom $\omega^{\omega}$ they matched with a similar number of controls with the intention of determining whether there 3 . appeared a hysterical predilection in those affected ir as compared with the controls. The results were $f$ largely negative and had not been published when $\underset{\mathcal{~}}{ }$ Compston et al. (1970) criticized their report in a letter to the British Medical Journal.

\section{Conclusions}

Those of the Medical Staff of the Royal Free $\vec{z}$ Hospital who witnessed the epidemic in 1955 were firmly of the conclusion that they were dealing with $\stackrel{\Phi}{3}$ an organic disease complicated by encephalemyelitis in which myalgia was a dominant feature. Objective evidence of brain stem and spinal co involvement was observed. The term 'epidem neuromyasthenia' does not appear in any way to be. satisfactory name for the disease seen at the Royal Free Hospital and described in previous and subsequent outbreaks.

\section{References}

Compston, N., Dimsdale, H., Ramsay, A.M. \& Richardson, A.T. (1970) Epidemic malaise. British Medical Journal, 1, 362.

Crowley, N., Nelson, M. \& Stovin, S. (1957) Royal Free epidemic. Journal of Hygiene. Cambridge, 55, 102.

MCEvedY, C.P. \& BEARD, A.W. (1970) Royal Free epidemic of 1955: a reconsideration. British Medical Journal, 1, 7. McEvedy, C.P. \& BEARD, A.W. (1970) Concept of benign $\delta$ myalgic encephalomyelitis. British Medical Journal, 1, 11. Medical Staff of the Royal Free Hospital (1957) An outbreak of encephalomyelitis in the Royal Free Hospital 윽 Group, London, in 1955. British Medical Journal, 2, 895. RAMSAY, A.M. (1978) 'Epidemic neuromyasthenia' 1955-
1978. Postgraduate Medical Journal, 54, 718.

Ramsay, A.M. \& O'Sullivan, E. (1956) Encephalomyelitis N simulating poliomyelitis. Lancet, i, 761 .

Richardson, A.T. (1956) Some aspects of the Royal Free $\mathrm{N}$ Epidemic. Annals of Physical Medicine, 3, 81. 